\title{
The role of echocardiography in transcatheter aortic valve implantation
}

\author{
Toshinari Onishi ${ }^{1}$, Kaoruko Sengoku ${ }^{1}$, Yasuhiro Ichibori ${ }^{1}$, Isamu Mizote ${ }^{1}$, Koichi Maeda ${ }^{2}$, Toru Kuratani ${ }^{2}$, \\ Yoshiki Sawa $^{2}$, Yasushi Sakata ${ }^{1}$ \\ ${ }^{1}$ Department of Cardiovascular Medicine, ${ }^{2}$ Department of Cardiovascular Surgery, Osaka University Graduate School of Medicine, Suita, Japan \\ Contributions: (I) Conception and design: T Onishi, Y Sakata; (II) Administrative support: None; (III) Provision of study materials or patients: All \\ authors; (IV) Collection and assembly of data: T Onishi; (V) Data analysis and interpretation: T Onishi, Y Sakata; (VI) Manuscript writing: All \\ authors; (VII) Final approval of manuscript: All authors. \\ Correspondence to: Toshinari Onishi. Department of Cardiovascular Medicine, Osaka University Graduate School of Medicine, 2-2 Yamadaoka, Suita, \\ Osaka 565-0871, Japan. Email: onishit@cardiology.med.osaka-u.ac.jp.
}

\begin{abstract}
Transcatheter aortic valve implantation (TAVI) is an effective and less invasive treatment for the increasing population of individuals with severe aortic stenosis (AS). Echocardiography is crucial in the assessment of AS patients from pre- to post-procedure. Transthoracic echocardiography (TTE) may be used to assess patient suitability for TAVI, as well as evaluate the severity of AS, the aortic valve complex, aortic valve morphology, mitral regurgitation (MR), and left ventricular function. Transesophageal echocardiography (TEE) is usually used as an intra-procedural monitoring tool to provide feedback during the procedure, to assess prosthetic valve function, and to detect complications rapidly before and after balloon aortic valvuloplasty (BAV) or transcatheter heart valve (THV) deployment. In this review, the role of echocardiography in the pre-, intra-, and post-TAVI procedure periods is described in detail.
\end{abstract}

Keywords: Echocardiography; aortic stenosis (AS); transcatheter aortic valve implantation (TAVI)

Submitted Oct 22, 2017. Accepted for publication Jan 11, 2018.

doi: $10.21037 / \mathrm{cdt} .2018 .01 .06$

View this article at: http://dx.doi.org/10.21037/cdt.2018.01.06

\section{Introduction}

Aortic stenosis (AS) has been the most common valvular heart disease in the past decades (1). As a result of the PARTNER trial (2), transcatheter aortic valve implantation (TAVI) provides an effective and less invasive alternative to surgical aortic valve replacement (SAVR) for inoperable and high-risk patients with symptomatic AS (3). As the use of TAVI for intermediate-risk patients has also been found acceptable based on a recent clinical trial (4), indications for TAVI may be increasing in the future.

Echocardiography is vital in the assessment of AS patients from pre- to post-procedure for TAVI. Initial evaluation is mainly performed by transthoracic echocardiography (TTE) to assess patient suitability for TAVI, including the severity of AS, assessment of the aortic valve complex and aortic valve morphology, and quantification of mitral regurgitation (MR) and left ventricular function. Transesophageal echocardiography (TEE) is usually used as an intra-procedural monitoring tool to provide feedback during the procedure; to assess prosthetic function; and to detect complications, such as aortic regurgitation (AR), mitral valve damage, pericardial effusion, ventricular dysfunction, and aortic rupture or dissection, before and after balloon aortic valvuloplasty (BAV) or transcatheter heart valve (THV) deployment. In the post-procedure period, TTE should be considered as the primary imaging modality for the assessment of prosthetic valve function. Since THV involves prosthetic valves made from cow or pig tissues, late degeneration, such as stenosis or regurgitation, infective endocarditis (IE), and thrombosis may occur. Valve durability and dysfunction may become critical problems over time.

This review describes the role of echocardiography in 
the pre-, intra-, and post-procedure periods.

\section{Pre-procedural echocardiography in TAVI}

The pre-procedural echocardiographic evaluation for TAVI is mainly performed with TTE to assess valvular and ventricular morphology and function. The general approaches for assessing AS, AR, and MR are published by the American Society of Echocardiography (5).

\section{Severity of $A S$}

Current indications for aortic valve replacement (AVR), including TAVI, are based on the severity of AS in symptomatic patients, including patients with evidence of left ventricular compromise [left ventricular ejection fraction (LVEF) $<50 \%]$ (6). According to the guidelines, severe AS is defined as a calculated aortic valve area (AVA) of $\leq 1.0 \mathrm{~cm}^{2}\left(\leq 0.6 \mathrm{~cm}^{2} / \mathrm{m}^{2}\right)$, a peak transvalvular velocity of $\geq 4 \mathrm{~m} / \mathrm{s}$, or a mean aortic valve gradient of $\geq 40 \mathrm{mmHg}$ (6).

Although most patients with severe AS exhibit a high transvalvular gradient, some patients with a lower gradient (peak transvalvular velocity of $<4 \mathrm{~m} / \mathrm{s}$ ) but severe AS are found in the situation of low aortic transvalvular flow (stroke volume index $<35 \mathrm{~mL} / \mathrm{m}^{2}$ ) either with an impaired LVEF (classical low-flow low-gradient severe AS) or with a normal LVEF (paradoxical low-flow low-gradient severe AS) (7). While the former is primarily due to low stroke volume (SV) with overt left ventricular (LV) systolic dysfunction, the latter is mainly due to low SV with a smaller $\mathrm{LV}$ and a restrictive physiology (8). Thus, it is important to distinguish between true severe AS and pseudo-severe AS. Dobutamine stress echocardiography has been useful in differentiating between them (it is negative in the pseudo-severe condition) (5) and predicting the risk of adverse events in the severe condition $(9,10)$. Projected AVA has been proposed to standardize the calculation of AVA at a transvalvular normal flow rate of $250 \mathrm{~mL} / \mathrm{s}$ and distinguish between them $(10,11)$. This concept is theoretically attractive because it offers a rational solution to the common problem of achieving different. Although it is controversial whether the prognosis of paradoxical low-flow low-gradient severe AS is better than that of low-flow high-gradient severe AS $(8,12)$, Herrmann et al. demonstrated improved survival in patients with lowflow low-gradient severe AS following TAVI compared with medical therapy at 2 years $(56.5 \%$ vs. $76.9 \%)$ in a sub-analysis of the PARTNER trial (13).

Beyond these pathophysiological findings, special attention to the following points needs to be paid to this inconsistency in grading the severity of AS. At first, multiple measurements with TTE are made using different acoustic windows to measure the maximum velocity and highest mean gradient across the stenotic valve. Thaden et al. showed that in 100 consecutive patients with severe AS, the right parasternal window was superior for identifying maximal velocity. When sampling maximal velocity only from the apical window, nearly a quarter of patients were misclassified, of whom two-thirds were underestimated as moderate AS and one-third were misclassified from highgradient severe AS to low-gradient severe AS (14).

Measurement of the LV outflow tract (LVOT) dimensions is measured in mid-systole at the same time in the cardiac cycle as the maximum LVOT velocity just below the insertion of the aortic valve leaflets (15), and it may have relatively high inter-observer variability (16). Furthermore, since the LVOT is elliptical in patients with AS, and measurement using two-dimensional (2D)-TTE is often the shortest dimension, stroke volume and calculated AVA by the continuity equation may be underestimated (17). When three-dimensional (3D)-TTE reveals the elliptical LVOT and its area is measured, AS grading accuracy may be improved (18).

\section{Aortic valve complex}

Assessment of the aortic valve complex, which includes the LVOT, aortic annulus, aortic valve cusps, sinus of valsalva, sinotubular junction, and position of the coronary arteries, is important in deciding the size of the THV to avoid complications such as paravalvular regurgitation, annular rupture, or coronary artery occlusion (19-22).

The most important approach for THV sizing is measurement of the aortic annulus, which is a "virtual ring" at the level of the hinge point of the three aortic valve cusps (23). Since the annulus is often asymmetric and oval, annular diameters should be largest in the coronal plane and shortest in the sagittal plane (24-26) (Figure 1). The conventional measurement for annular size has been mid-systolic diameter in the long axis plane (parasternal for TTE, mid-esophageal for TEE) that bisects the largest dimension of the aortic annulus (27). Recently, the annular diameter calculated from the annular perimeters or area measurements delivered by multiple detector computed tomography (MDCT) has become the gold standard $(28,29)$. In general, the annular diameter measured by 2D-TTE has been shown to be smaller than that by $2 \mathrm{D}$-TEE, and aortic annular area by 


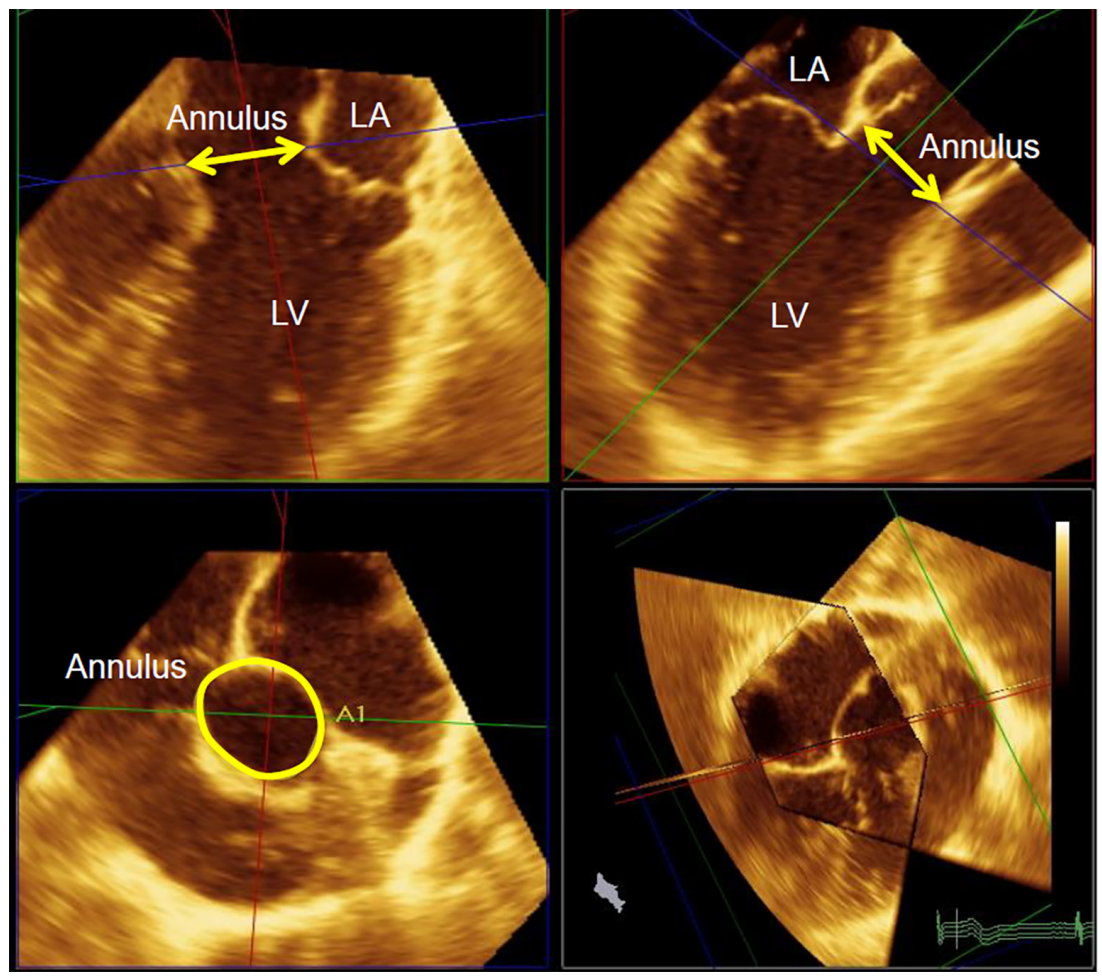

Figure $13 \mathrm{D}$ transesophageal echocardiography assessing asymmetric and oval-shaped aortic annulus. LV, left ventricle; LA, left atrium.

2D-TEE to be underestimated than that by $3 \mathrm{D}-\mathrm{TEE}$ or 3D-TTE in comparison to MDCT (30-32). Either by echocardiography or by MDCT, the following annular measurements were performed: perimeter, area, and orthogonal maximum and minimum diameters. Average diameter was calculated from perimeter (perimeter $/ \pi$ ) and area $[2 \times \sqrt{(\text { area } / \pi)}]$. Mean diameter was calculated as the average of the maximum diameter and minimum diameter. Oversizing of the THV can result in aortic annular rupture, and undersizing can lead to increased paravalvular leak (PVL), valve 'pop-out' or migration (20). For each type of THV, the recommended device/annulus oversizing is required to prevent PVL calculated from the aortic basal ring perimeter, area, or mean diameter.

Coronary obstruction is related to the displacement of a calcified native aortic valve leaflet into the coronary ostium. Ribeiro et al. reported in the recent meta-analysis that it occurred more frequently in the left coronary artery (83\%), the height of the left main above the annulus was on average $10.3 \mathrm{~mm}$ (range, 7 to $>12 \mathrm{~mm}$ ), and approximately $60 \%$ of occlusions occurred with a coronary height $>10 \mathrm{~mm}$ (22).

Calcification within the aortic complex should be noted because when it involves the LVOT, aortic leaflets, or proximal root, it is predictive of postprocedural AR $(33,34)$, annular rupture $(19,35)$, root perforation, aortic wall hematoma, and dissection (36). Calcifications around the coronary ostia may lead to coronary obstruction (37).

\section{Aortic valve morphology}

Bicuspid aortic valve disease is challenging for TAVI, and has been so far an exclusion criterion in randomized TAVI trials (2). Although there are some reports of TAVI being successfully performed for a bicuspid valve $(38,39)$, there are some issues due to asymmetry of the aortic valve opening, including greater severity of post-TAVI PVL and higher mortality and incidence of device malposition $(39,40)$. In the multicenter retrospective analysis of 139 patients with bicuspid valve, Mylotte et al. reported a procedural mortality of $3.6 \%$, incidences of THV embolization and conversion to surgery of $2.2 \%$, and 1 -year mortality rate of $17.5 \%$. The incidence of PVL was $28.4 \%$ (more than mild) and $8 \%$ (more than moderate). There was no difference in PVL between self-expanding and balloon-expandable valves $(\mathrm{P}=0.99)$ (40). TAVI for bicuspid valve remains controversial. 


\section{$M R$}

Significant MR is frequent in patients with severe AS. Prevalence is up to $74 \%$ of elderly candidates for SAVR or TAVI (41). Thus, accurate evaluation for MR on preprocedural echocardiography is important.

MR can be often classified into primary degenerative and secondary functional subsets. Nombela-Franco et al. demonstrated in their review that MR severity improves after TAVI, especially in patients with LV dysfunction and functional MR (41). Other studies also suggested that outcomes were better with functional than with degenerative MR $(42,43)$. However, Chakravarty et al. evaluated the impact of MR on outcomes after TAVI by performing a metaanalysis of 8 studies involving 8,927 patients, and showed that the increased mortality associated with moderate-tosevere MR was not influenced by the cause of MR [functional or degenerative MR; RR 0.90, 95\% confidence interval (CI): 0.62 to $1.30, \mathrm{P}=0.56]$. They concluded that baseline moderate-to-severe MR and significant residual MR after TAVI are associated with an increase in mortality after TAVI (44). The management of patients with severe AS and concomitant $\mathrm{MR}$ remains challenging.

\section{Left ventricular function}

Successful TAVI is associated with a significant improvement in LV systolic and diastolic function (45-47).

Webb et al. demonstrated that LVEF increased after TAVI from a mean of $53 \%$ to $57 \%(\mathrm{P}<0.0001)$ within days and was sustained up to 1 year in 50 patients referred for TAVI. In particular, an LVEF $\leq 40 \%$ at baseline was documented in $21 \%$ of patients before successful valve implantation, falling to $12 \%, 13 \%, 0 \%$, and $6 \%$ prevalence at discharge and 1, 6, and 12 months, respectively (45). Patients with severe AS and reduced LVEF have a poor prognosis with conservative therapy but high operative mortality when treated surgically. Even in patients with severe $\mathrm{LV}$ dysfunction (LVEF $\leq 35 \%$ ), AVR is associated with a large mortality benefit $(48,49)$. Passeri et al. demonstrated that in 342 inoperable patients for SAVR undergoing TAVI or standard therapy, baseline LV dysfunction did not affect survival after TAVI but was associated with increased cardiac mortality at 1 year with standard therapy $[59.3 \% \mathrm{vs}$. $45.8 \%$ with normal LVEF; hazard ratio $(\mathrm{HR})=1.71$ (95\% CI: 1.08 to 2.71); $\mathrm{P}=0.02$ ]. TAVI improves survival in patients with severe $\mathrm{LV}$ dysfunction (50).

Improvement of LV diastolic function should play an important role in reducing postoperative morbidity and mortality. Gonçalves et al. reported acute improvement of LV diastolic function immediately after successful TAVI in a group of 61 AS patients with preserved LVEF. They showed a reduction in hemodynamic invasive LV enddiastolic pressure a few minutes after TAVI and a significant improvement in the $L V$ restrictive filling pattern without a significant decrease in E/E ratio (46). In 135 patients with successful TAVI, Vizzardi et al. demonstrated that LV mass index decreased from $191 \pm 58$ (baseline) to $132 \pm 30 \mathrm{~g} / \mathrm{m}^{2}$ (6 months after TAVI) $(\mathrm{P}<0.001)$ and that 97 patients $(72 \%)$ showed improvement in $\mathrm{LV}$ diastolic filling pattern (47).

\section{Left ventricular structure}

Combined valvular and subvalvular LV outflow obstruction can be the result of either independent processes of valvular AS and coincident hypertrophic obstructive cardiomyopathy (HOCM), or the development of subvalvular LV outflow obstruction as a result of LV hypertrophy that occurs as a result of the increased afterload of AS (51). Echocardiography can adequately document the presence of obstructive flow pattern with the jet peaks late in systole (52) and specific echocardiographic parameters can guide interventionalist or surgeon whether TAVI can be performed without complication. Or, SAVR should be performed along with surgical myectomy to relieve LVOT obstruction post AVR (51).

\section{Intra-procedural echocardiography in TAVI}

TEE is usually used as an intra-procedural monitoring tool to provide feedback during the procedure, to assess the prosthetic valve function, and mainly to detect complications rapidly before and after BAV or THV deployment, although general anesthesia is required for TEE in elderly patients with severe AS and multiple comorbidities.

\section{Immediate pre-procedure}

Immediately before the procedure, TEE should be performed as a baseline morphological and hemodynamic assessment for TAVI to evaluate all four valves and four chambers, assessing chamber size and wall motion, and quantifying heart valve regurgitation. It is very important to assess MR at baseline because the severity of MR may change dramatically during the procedure due to mechanical 
compromise of the mitral apparatus by stiff wires, cannulae, LV dysfunction, increases in blood pressure, severe AR, systolic anterior motion following the abrupt reduction in afterload, or the THV itself (53).

\section{Wire insertion and position}

It is often difficult to pass a guidewire retrogradely through

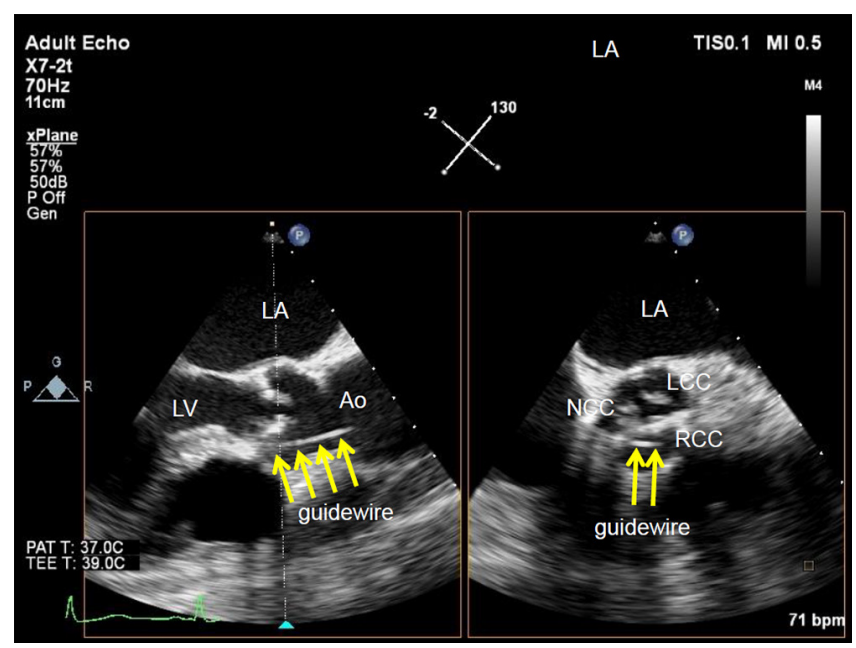

Figure 2 Wire insertion assisted by simultaneous multiplane imaging. Transesophageal echocardiography can demonstrate the location of a guidewire in the RCC of the aortic valve (yellow arrows), with long (left panel) and short axis views (right panel), using simultaneous multiplane imaging. LV, left ventricle; LA, left atrium; Ao, aorta; LCC, left coronary cusp; RCC, right coronary cusp; NCC, non-coronary cusp. the narrow aortic valve in patients with AS. This is a routine first step in TAVI, and TEE can provide the information on guidewire location, with simultaneous multiplane imaging (Figure 2).

2D- and 3D-TEE can easily visualize the position of the retrograde stiff wire with the coiled section of the tip at the apex in the LV for stability (Figure 3). Since wire injury or entanglement within the mitral apparatus may cause worsening of MR, TEE can help avoid it.

When the TAVI approach is done via a trans-apical approach, additional imaging is required. To ensure optimal location of the apical puncture, a cannulation site in the anterior apex is pushed by the surgeon's finger (Figure 4). When the guidewire or stiff wire is run through the "shortcut" in the LV, TEE should be performed to ensure avoiding the mitral valve apparatus (Figure 5).

\section{$B A V$}

BAV is often performed before TAVI to facilitate delivery of the THV and to expand the calcified aortic valve annulus after creating fractures of the calcified leaflets and increasing leaflet flexibility. Observation with TEE during and following BAV is important to assess the functional results of the dilation and to detect possible adverse events, including post-valvuloplasty AR, acute coronary occlusion, and cardiac tamponade.

\section{THV deployment}

To precisely position the THV is critically important. Although fluoroscopy plays a central role, TEE is an adjunct
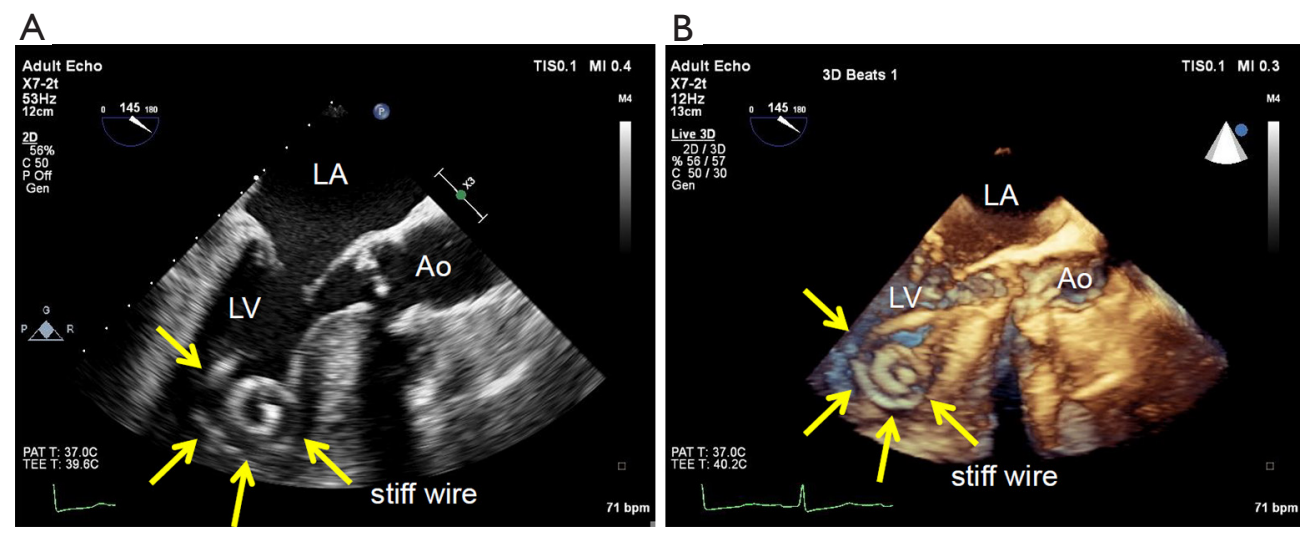

Figure 3 The optimal position of the retrograde stiff wire in the left ventricle. The retrograde stiff wire and the coiled section of the tip (yellow arrows) are appropriately positioned at the apex of the left ventricle. (A) 2D-TEE; (B) 3D-TEE. LV, left ventricle; LA, left atrium; Ao, aorta. 
imaging in confirming the correct position during the THV deployment. To perform TEE may improve procedural results mainly thanks to its rapid detection of complications. Currently, there are three types of THV, those are the balloonexpandable, self-expanding, and mechanically expanded THV. How to deploy them is technically different for each.

\section{Immediate post-THV deployment}

Immediately after THV deployment, TEE rapidly provides an accurate assessment of valve position, shape, leaflet

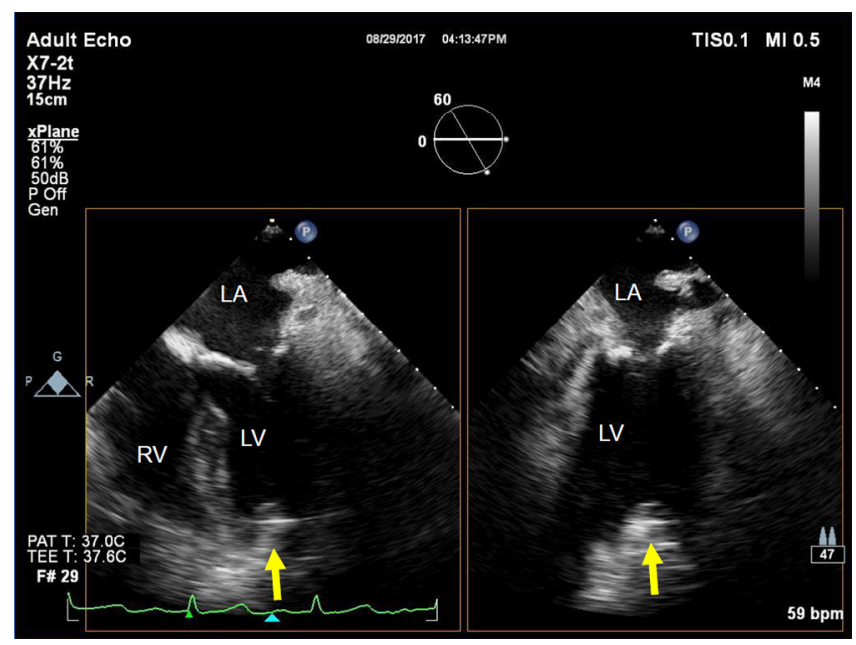

Figure 4 The optimal location of the apical puncture. The optimal location of the apical puncture is determined by visualizing the surgeon's fingers (yellow arrows) using transesophageal echocardiography. LV, left ventricle; LA, left atrium; RV, right ventricle. motion, maximal velocity, mean pressure gradient, and effective orifice area (EOA). Balloon post-dilation is usually needed when valve shape is too oval due to underexpansion and maximal velocity or mean pressure gradient is too high, measured by trans-gastric TEE view which is sometimes technically difficult to detect. Additionally, TEE can assess for complications, such as post-procedural AR, mitral valve damage, pericardial effusion, ventricular dysfunction, and aortic rupture or dissection.

\section{Complications}

\section{AR}

Both transvalvular and paravalvular AR may occur after THV deployment. Numerous studies have shown an association between post-procedural AR and increased short- and long-term mortality $(54,55)$.

Transvalvular AR is commonly associated with the stiff wire across the valve (Figure 6), and often improves after it is removed.

It is often difficult to evaluate the severity and location of paravalvular AR because the anatomy and physiology of regurgitant jets differ from those in conventional SAVR with a sewing ring. It is usually important to detect the true orifice of paravalvular AR to prevent the overestimation of AR severity using the long-axis view by rotating the TEE probe from medial to lateral, the short-axis view at the level of the aortic annulus, or simultaneous multiplane imaging (Figure 7). Deep gastric views can be also helpful in detecting AR, but jet area and length cannot be used for AR severity (56). Current guidelines state that a circumferential extent $<10 \%$ of paravalvular AR can be associated with mild,
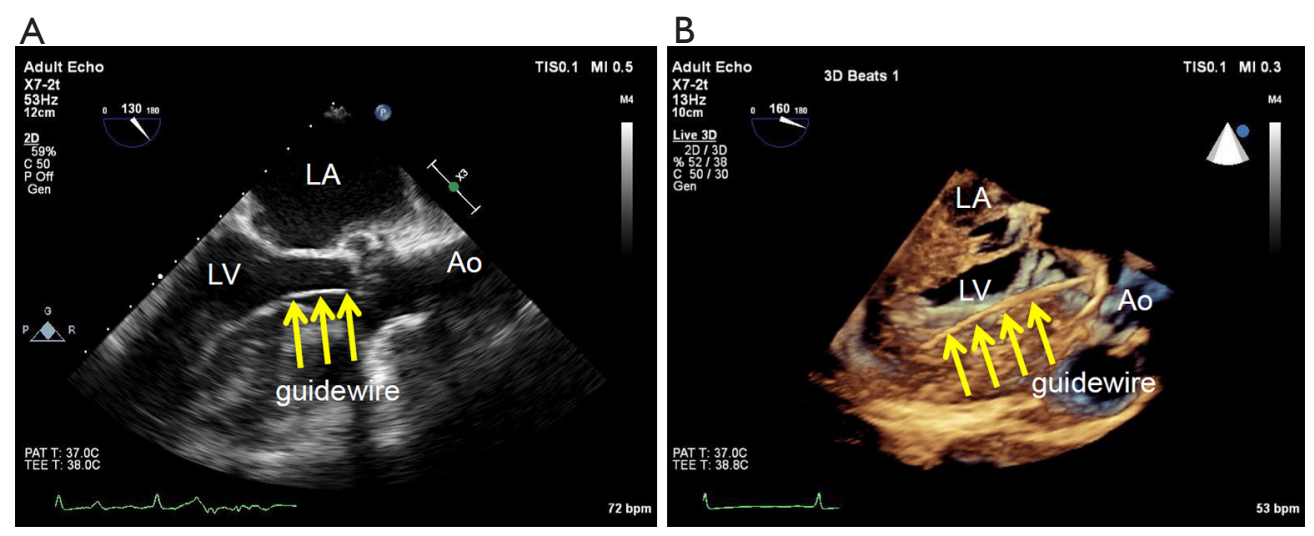

Figure 5 The optimal position of the guidewire or stiff wire. The guidewire or stiff wire (yellow arrows) for a transapical case is properly positioned across the aortic valve with no entanglement in the mitral apparatus. (A) 2D-TEE; (B) 3D-TEE. LV, left ventricle; LA, left atrium; Ao, aorta. 


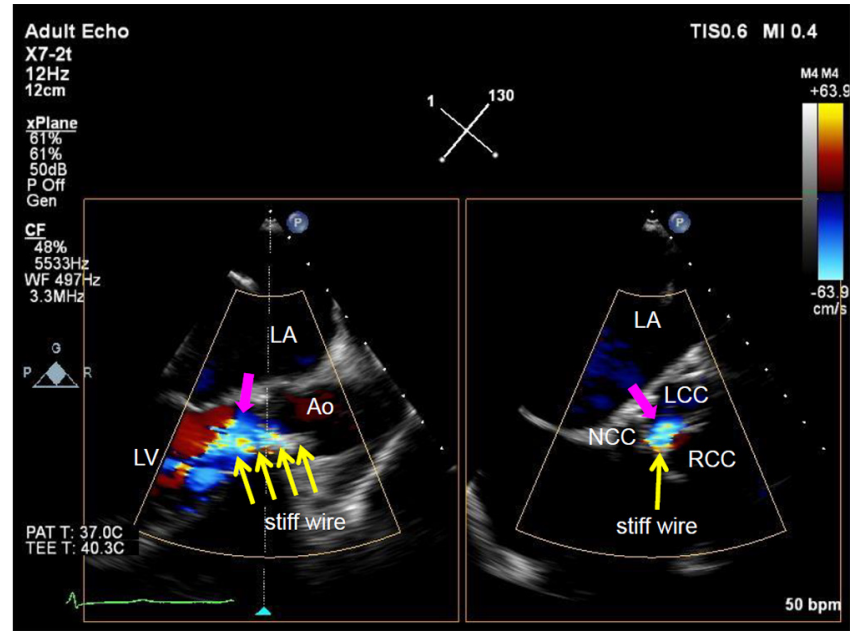

Figure 6 Transvalvular aortic regurgitation after transcatheter heart valve (THV) deployment. Transvalvular aortic regurgitation (pink arrow) occurrence due to location of the stiff wire (yellow arrows) across the valve after THV deployment. $\mathrm{LV}$, left ventricle; LA, left atrium; Ao, aorta; LCC, left coronary cusp; RCC, right coronary cusp; NCC, non-coronary cusp.

$10 \%$ to $20 \%$ with moderate, and $>20 \%$ with severe $(57)$ (Figure 8). However, paravalvular AR is commonly eccentric and can have multiple jets, and it is therefore uncertain how these jets should be summed (56).

There are three important factors in paravalvular AR: undersizing of the prosthesis, severity of aortic calcification, and prosthesis position in relation to the annulus (20). The first, undersizing of the prosthesis relative to the annulus size, is a common cause of paravalvular AR after TAVI. Détaint et al. demonstrated the effect of undersizing using the cover index [100x (prosthesis diameter - TEE annulus diameter)/ prosthesis diameter], and found this to be an independent predictor of moderate or severe AR post THV deployment [odds ratio 1.22; 95\% CI: $1.03-1.51, \mathrm{P}<0.02$ ] (21). The second, aortic valve calcification, also influences the severity of paravalvular AR post TAVI. Colli et al. demonstrated that the calcification score by TEE allowed prediction of the risk of paravalvular AR after TAVI (odds ratio 8.5 ; 95\% CI: $1.2-58.9$; $\mathrm{P}<0.0001$ ) (58). Finally, postTAVI AR is influenced by prosthesis position in relation to the annulus. When the THV is misplaced higher or lower, the skirt of the valve does not serve its function as an adequate seal around the annulus, resulting in AR. In cases of significant paravalvular AR, additional balloon dilatation or a second THV implantation is sometimes needed.
Mitral valve damage

Severe MR due to valvular perforation (Figure 9) or ruptured chordae might occur during the procedure. Frequent evaluation of the severity of MR and the anatomy of the mitral apparatus is always important.

\section{Pericardial effusion}

Pericardial effusion can indicate localized bleeding during the procedure. Whenever pericardial effusion is suddenly observed, evaluation for tamponade physiology and etiology, such as chamber perforation or aortic dissection, is required.

\section{Ventricular dysfunction}

Coronary obstruction due to displacement of the calcified native valve leaflets over the coronary ostia during the procedure can result in regional wall motion abnormalities. It is necessary to confirm regional or global wall motion abnormalities of the LV and RV, and to assess blood flow in the coronary ostia using color Doppler imaging.

\section{Aortic rupture or dissection}

Extensive annular calcification or prosthesis oversizing increases the risk of aortic rupture (Figure 10) or dissection after BAV or THV deployment. The aortic root and ascending aorta should be carefully examined to determine whether periaortic hematoma, aortic dissection, or rupture including ventricular septal defect or $\mathrm{LV}$ to left atrial shunt has occurred. They will likely cause massive bleeding and tamponade.

\section{Post-procedural echocardiography in TAVI}

Surveillance TTE should be considered as the primary imaging modality for the assessment of prosthetic valve function. The Valve Academic Research Consortium (VARC)-2 suggested the following schedule: immediately (before discharge) following THV implantation; 1, 6, and 12 months after implantation; and yearly thereafter (59). Additionally, an urgent TTE should be performed when a new murmur or new congestive heart failure symptoms appear in patients with THV.

In general, most surgical biological valves have limited durability and degenerate within 10-20 years (60). Since THV involves prosthetic valves made from cow or pig tissues, late degeneration may occur as the major 

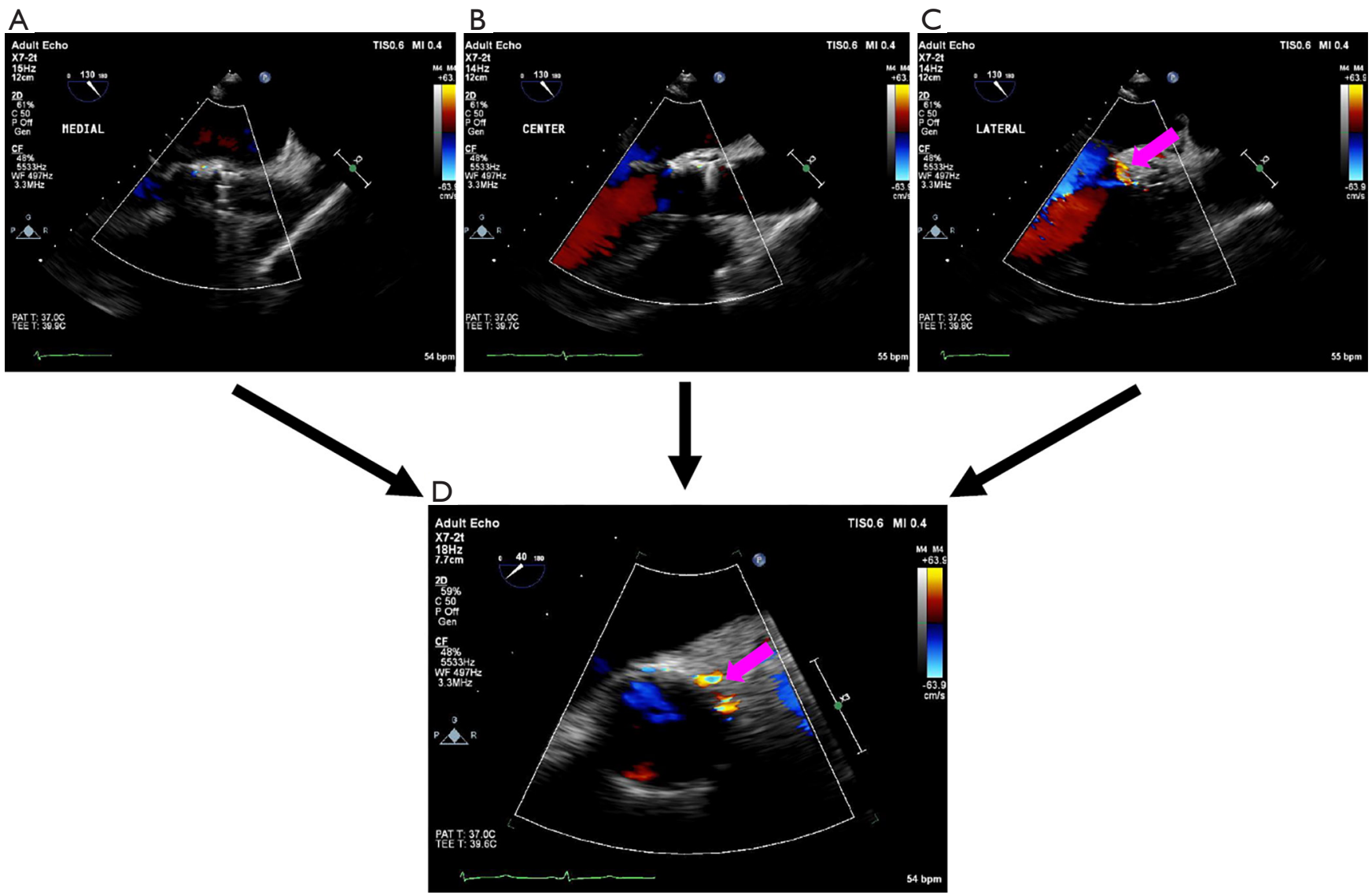

Figure 7 Detection of paravalvular aortic regurgitation (AR) after transcatheter heart valve (THV) deployment. Transesophageal echocardiography (TEE) can detect the true orifice of paravalvular AR (pink arrow) to prevent overestimation of AR severity using the longaxis view by rotating a TEE probe from the medial (A), to the center (B) to the lateral (C) side, and the short-axis view at the level of the aortic annulus (D).

complication.

\section{Prosthesis-patient mismatch}

Prosthesis-patient mismatch (PPM) occurs when the EOA of the implanted prosthesis is too small in relation to body size. The severity of PPM is graded using EOA indexed to body surface area (EOAi) $(56,59)$ with absence defined as $>0.85 \mathrm{~cm}^{2} / \mathrm{m}^{2}$, moderate as $0.65 \leq$ EOAi $<0.85 \mathrm{~cm}^{2} / \mathrm{m}^{2}$, and severe as $<0.65 \mathrm{~cm}^{2} / \mathrm{m}^{2}$. PPM affects $\mathrm{LV}$ mass regression (61) and long-term survival (62) after SAVR. In the PARTNER trial, Hahn et al. demonstrated that TAVI patients had less PPM than SAVR patients over 2 years (moderate 34\%, severe $23 \%$ for TAVI vs. $48 \%, 23 \%$ for SAVR, $\mathrm{P}=0.019$ ) and PPM in TAVI patients is associated with lower mortality (63). Pibarot et al. demonstrated in a post hoc analysis of the
PARTNER cohort A trial that the incidence of PPM was higher in SAVR than in TAVI (28\% vs. $20 \%)$ with a more significant difference when dealing with small aortic annulus diameters (<20 mm) (34\% vs. 19\%) (64). They concluded that TAVI might be preferable to SAVR in patients with a small aortic annulus who are susceptible to PPM to avoid its adverse impact on LV mass regression and survival (64).

\section{Structural valve deterioration}

Structural valve deterioration (SVD) is defined as any change in function of a THV resulting from any valve abnormality, including THV stenosis and regurgitation exclusive of infection or thrombosis. In their study of 8,914 TAVI patients, Foroutan et al. concluded that SVD is probably an infrequent event within the first 5 years, and 

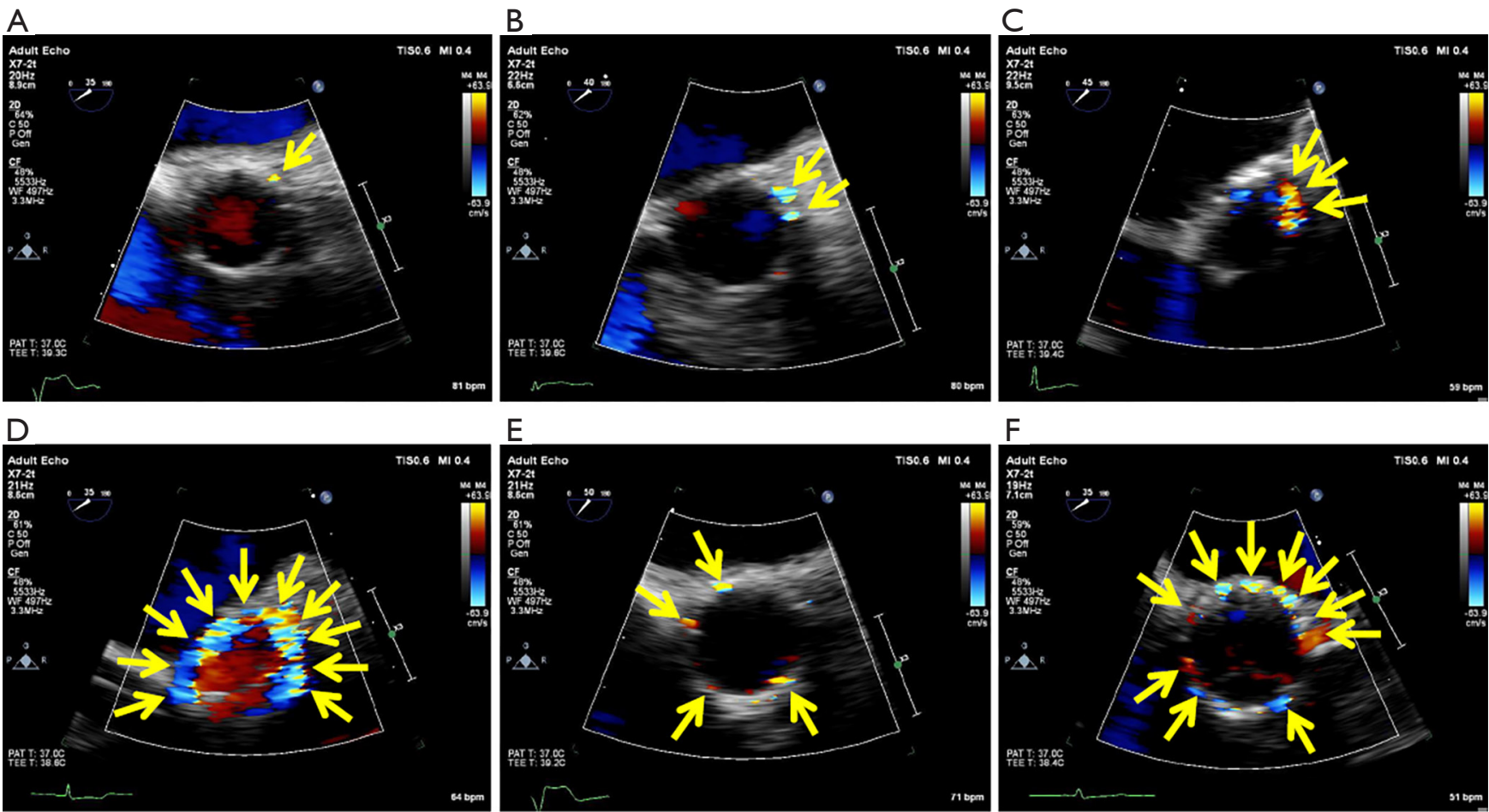

Figure 8 Severity of paravalvular aortic regurgitation (AR) after transcatheter aortic valve deployment. Although the severity of paravalvular AR depends on the circumferential extent, it is uncertain how these jets should be summed due to eccentric and multiple jets (yellow arrows). (A) trivial AR; (B) mild AR; (C) moderate AR; (D) severe AR; (E) trivial AR; (F) mild AR.
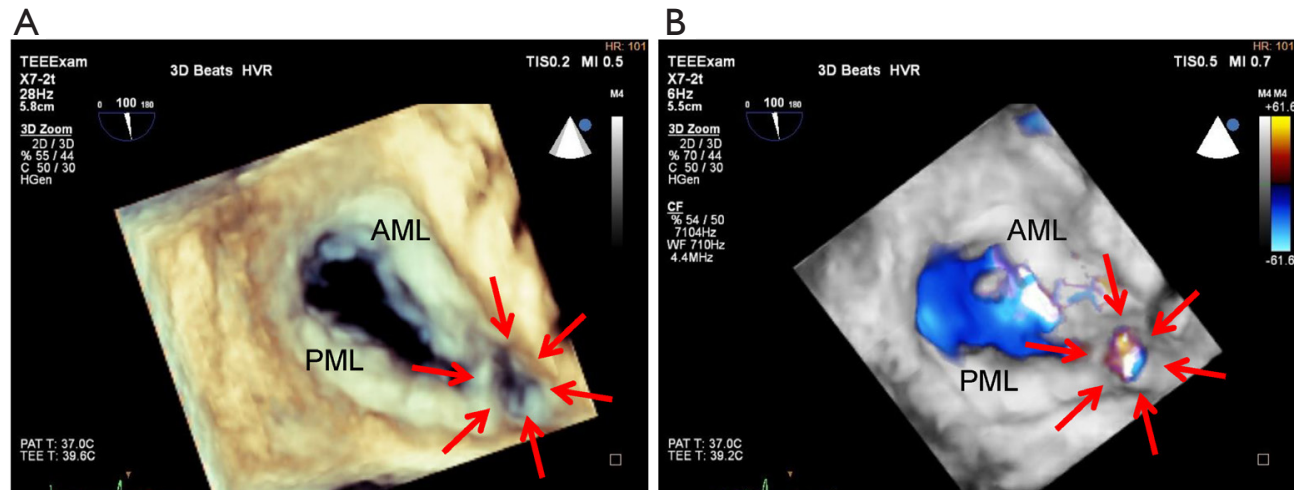

Figure 9 Acute mitral regurgitation due to mitral leaflet perforation. Acute mitral regurgitation due to mitral leaflet perforation close to the posterior commissure (red arrows) occurred after transcatheter heart valve (THV) deployment because the THV might be placed with wire entanglement in the mitral apparatus. (A) 3D imaging; (B) 3D color Doppler imaging. AML, anterior mitral leaflet; PML, posterior mitral leaflet.

longer follow-up (>10 years) is required (65).

\section{THV stenosis or regurgitation}

THV stenosis may sometimes occur as a result of calcification, or less frequently due to pannus (Figure 11). If the peak aortic velocity is $>3.0 \mathrm{~m} / \mathrm{s}$ or mean gradient is $>20 \mathrm{mmHg}$, THV stenosis can be suspected (Table 1) $(56,59)$. Although peak velocity and mean gradient are flowdependent parameters, Doppler velocity index (DVI) is 
independent of flow and the size of the inserted valve. Thus, DVI can be more useful in detecting valve dysfunction. A normal DVI indicates basically normal prosthetic valve function. When the EOAi is low in the setting of a normal DVI, the patient is considered to have a PPM (59). THV regurgitation may occur as a consequence of wear and tear, such as leaflet prolapse (Figure 12) or calcification. A transcatheter valve-in-valve procedure may be chosen for the treatment of THV stenosis and regurgitation.

\section{IE}

IE is a serious complication after TAVI as well as after SAVR (Figure 13). From a large multicenter registry of 7,944 patients, Amat-Santos et al. reported an incidence of IE at 1 year following TAVI of $0.5 \%$ with a median time from implantation of 6 months (66).

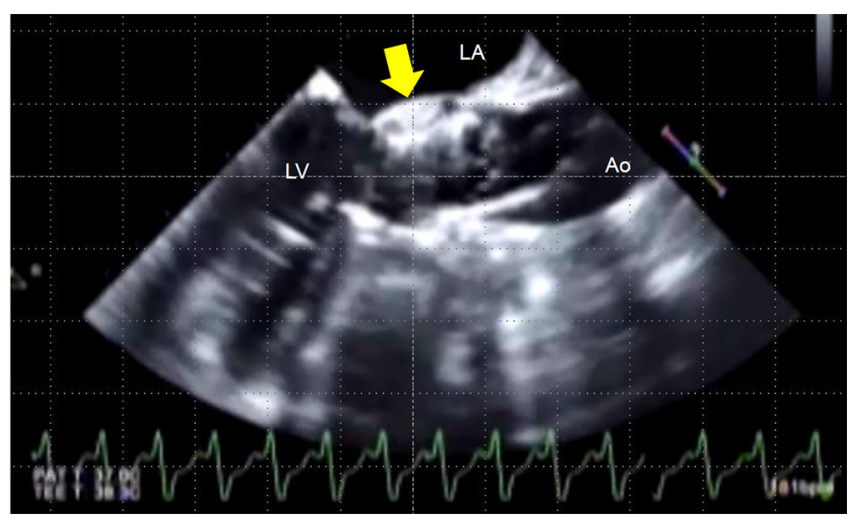

Figure 10 Aortic root rupture with periaortic hematoma. Aortic root rupture with periaortic hematoma (yellow arrow) after transcatheter heart valve deployment was observed in this case. LV, left ventricle; LA, left atrium; Ao, aorta.

\section{Clinical \& subclinical THV thrombosis}

Recently, hypoattenuated leaflet thickening (HALT) and reduced leaflet motion (RELM) of bioprosthetic aortic valves associated with normal transvalvular gradients have been reported as affecting THV. The prevalence of this finding ranged from $4 \%$ to $20 \%$ (67-69). Latib et al. first

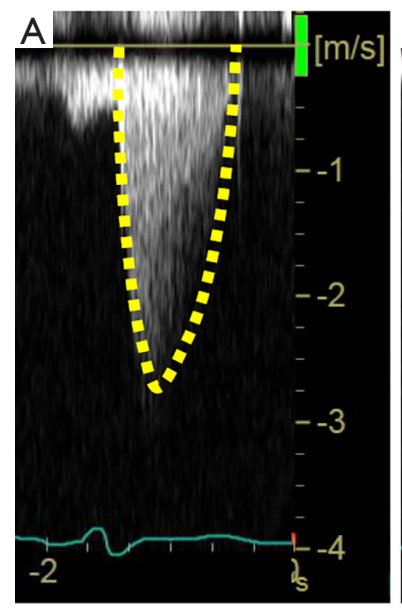

One week after THV deployment

Peak velocity $=2.9 \mathrm{~m} / \mathrm{s}$ Mean gradient $=21 \mathrm{mmHg}$ $\mathrm{DVI}=0.4$ $\mathrm{EOA}=1.7 \mathrm{~cm}^{2}$ $\mathrm{EOAi}=1.0 \mathrm{~cm}^{2} / \mathrm{m}^{2}$

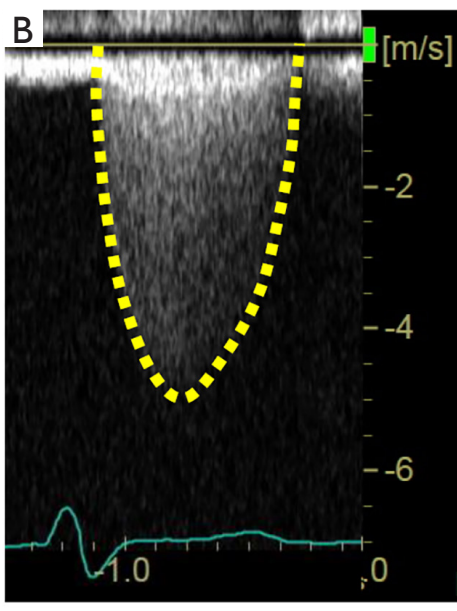

2 years after THV deployment Peak velocity $=4.9 \mathrm{~m} / \mathrm{s}$ Mean gradient $=62 \mathrm{mmHg}$ $\mathrm{DVI}=0.2$ $\mathrm{EOA}=0.5 \mathrm{~cm}^{2}$ EOAi $=0.3 \mathrm{~cm}^{2} / \mathrm{m}^{2}$
Figure 11 Transcatheter heart valve (THV) stenosis. Continuous wave Doppler velocity of the aortic valve in a patient with THV stenosis 1 week (A) and 2 years (B) after THV deployment. The peak aortic velocity, mean gradient, Doppler velocity index (DVI), effective orifice area (EOA), and EOA indexed to body surface area (EOAi) from $2.9 \mathrm{~m} / \mathrm{s}, 21 \mathrm{mmHg}, 0.4,1.7 \mathrm{~cm}^{2}$ and $1.0 \mathrm{~cm}^{2} / \mathrm{m}^{2}$ to $4.9 \mathrm{~m} / \mathrm{s}, 62 \mathrm{mmHg}, 0.2,0.5 \mathrm{~cm}^{2}$ and $0.3 \mathrm{~cm}^{2} / \mathrm{m}^{2}$.

Table 1 Transcatheter heart valve (THV) stenosis

\begin{tabular}{lccc}
\hline & & \multicolumn{2}{c}{ Prosthetic aortic valve stenosis } \\
\cline { 2 - 4 } Parameter & Normal & Mild stenosis & Moderate/severe stenosis \\
\hline Peak velocity $(\mathrm{m} / \mathrm{s})$ & $<3$ & $3-4$ & $>4$ \\
Mean gradient $(\mathrm{mmHg})$ & $<20$ & $20-40$ & $>40$ \\
Doppler velocity index & $>0.35$ & $0.35-0.25$ & $<0.25$ \\
Effective orifice area $\left(\mathrm{BSA}>1.6 \mathrm{~cm}^{2}\right)$ & $>1.1$ & $1.1-0.8$ & $<0.8$ \\
Effective orifice area $\left(\mathrm{BSA}<1.6 \mathrm{~cm}^{2}\right)$ & $>0.9$ & $0.9-0.6$ & $<0.6$ \\
\hline
\end{tabular}

\footnotetext{
${ }^{\dagger}$, for left ventricular outflow tract (LVOT) $>2.5 \mathrm{~cm}$, significant stenosis criteria is $<0.20$. BSA, body surface area.
} 

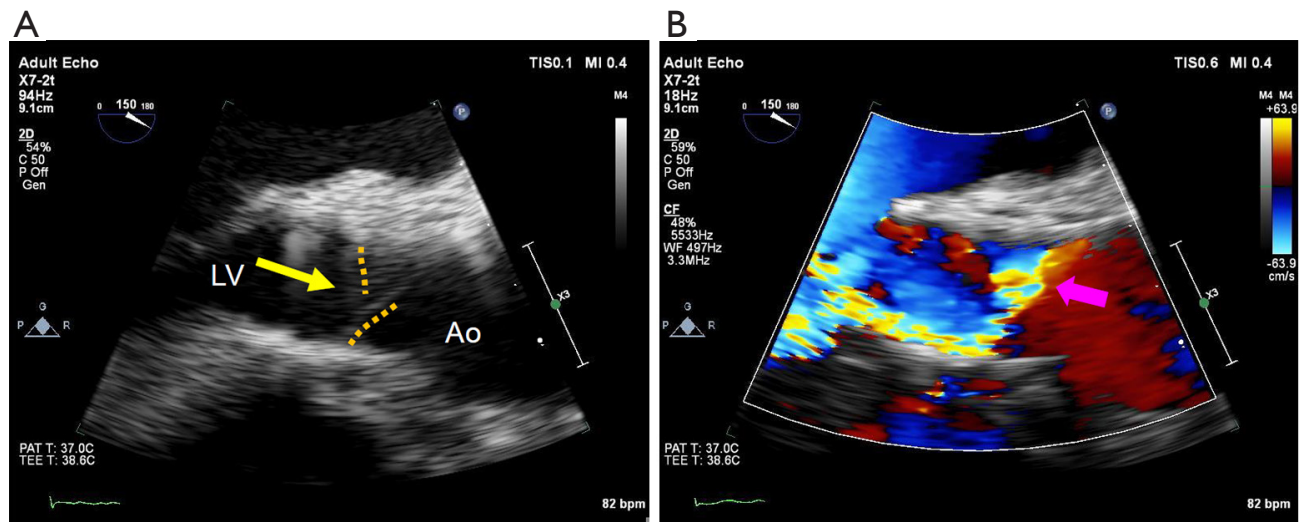

Figure 12 Transcatheter heart valve (THV) regurgitation. THV leaflet prolapse (orange dotted lines, yellow arrow) occurred 5 years after THV deployment (A). Severe THV regurgitation (pink arrow) could be observed (B). LV, left ventricle; Ao, aorta.

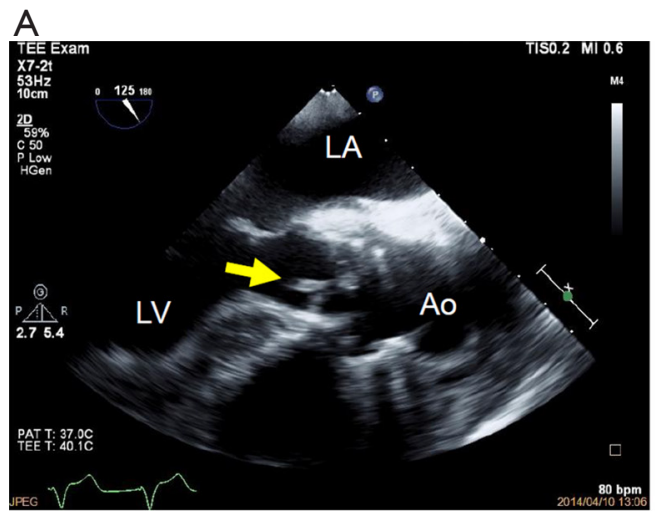

B

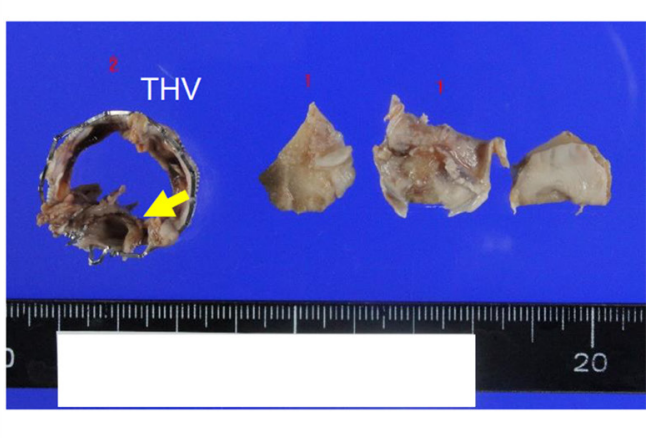

Figure 13 Infective endocarditis. Two months after transcatheter heart valve (THV) deployment, the patient presented with malaise, breathlessness and elevated markers of infection. TEE revealed small mobile echogenic masses attached to the THV leaflet (A). In the surgical findings, vegetation (yellow arrow) was identified on the valve leaflets and THV stent frame (B). LV, left ventricle; LA, left atrium; Ao, aorta; THV, transcatheter heart valve.

reported the treatment and clinical outcomes of THV thrombosis from a multicenter registry (69). The overall incidence of THV thrombosis was $0.6 \% \quad(n=26)$ out of 4,266 patients. The most common clinical presentation was exertional dyspnea $(n=17 ; 65 \%)$, whereas $8(31 \%)$ patients had no worsening symptoms. Echocardiography detected a markedly elevated mean aortic valve pressure gradient, presence of thickened leaflets or thrombotic apposition of leaflets in $20(77 \%)$ and a thrombotic mass on the leaflets in the remaining $6(23 \%)$ patients. Of 26 patients, $23(88 \%)$ were successfully treated with anticoagulation; two patients underwent a transcatheter valve-in-valve procedure and one patient underwent SAVR (69).

\section{Conclusions}

TAVI provides an effective, less-invasive alternative to SAVR for an increasing population of individuals with severe AS. TTE may be used to assess patient suitability for TAVI, and TEE can detect complications rapidly during TAVI. After THV deployment, TTE can evaluate prosthetic valve function. Echocardiography always serves an important function in the assessment of AS patients in any situation, from pre- to post-procedure for TAVI.

\section{Acknowledgements}

The authors thank Drs. Kei Torikai, Takashi Mukai, 
Masaki Tsuda for referring patients. We are also grateful for the excellent technical assistance provided by Ms. Keiko Katsuki, Ms. Yumiko Morimoto, Ms. Mariko Fujita.

\section{Footnote}

Conflicts of Interest: The authors have no conflicts of interest to declare.

\section{References}

1. Osnabrugge RL, Mylotte D, Head SJ, et al. Aortic stenosis in the elderly: disease prevalence and number of candidates for transcatheter aortic valve replacement: a meta-analysis and modeling study. J Am Coll Cardiol 2013;62:1002-12.

2. Leon MB, Smith CR, Mack M, et al. Transcatheter aorticvalve implantation for aortic stenosis in patients who cannot undergo surgery. N Engl J Med 2010;363:1597-607.

3. Joint Task Force on the Management of Valvular Heart Disease of the European Society of Cardiology (ESC); European Association for Cardio-Thoracic Surgery (EACTS), Vahanian A, et al. Guidelines on the management of valvular heart disease (version 2012). Eur Heart J 2012;33:2451-96.

4. Leon MB, Smith CR, Mack MJ, et al. Transcatheter or Surgical Aortic-Valve Replacement in Intermediate-Risk Patients. N Engl J Med 2016;374:1609-20.

5. Baumgartner H, Hung J, Bermejo J, et al. Echocardiographic assessment of valve stenosis: EAE/ ASE recommendations for clinical practice. J Am Soc Echocardiogr 2009;22:1-23; quiz 101-2.

6. Nishimura RA, Otto CM, Bonow RO, et al. 2014 AHA/ ACC Guideline for the Management of Patients With Valvular Heart Disease: executive summary: a report of the American College of Cardiology/American Heart Association Task Force on Practice Guidelines. Circulation 2014;129:2440-92.

7. Pibarot P, Dumesnil JG. Low-flow, low-gradient aortic stenosis with normal and depressed left ventricular ejection fraction. J Am Coll Cardiol 2012;60:1845-53.

8. Hachicha Z, Dumesnil JG, Bogaty P, et al. Paradoxical lowflow, low-gradient severe aortic stenosis despite preserved ejection fraction is associated with higher afterload and reduced survival. Circulation 2007;115:2856-64.

9. Grayburn PA. Assessment of low-gradient aortic stenosis with dobutamine. Circulation 2006;113:604-6.

10. Clavel MA, Ennezat PV, Marechaux S, et al. Stress echocardiography to assess stenosis severity and predict outcome in patients with paradoxical low-flow, lowgradient aortic stenosis and preserved LVEF. JACC Cardiovasc Imaging 2013;6:175-83.

11. Blais C, Burwash IG, Mundigler G, et al. Projected valve area at normal flow rate improves the assessment of stenosis severity in patients with low-flow, low-gradient aortic stenosis: the multicenter TOPAS (Truly or Pseudo-Severe Aortic Stenosis) study. Circulation 2006;113:711-21.

12. Yamashita E, Takeuchi M, Seo Y, et al. Prognostic value of paradoxical low-gradient severe aortic stenosis in Japan: Japanese Multicenter Aortic Stenosis Study, Retrospective (JUST-R) Registry. J Cardiol 2015;65:360-8.

13. Herrmann HC, Pibarot P, Hueter I, et al. Predictors of mortality and outcomes of therapy in low-flow severe aortic stenosis: a Placement of Aortic Transcatheter Valves (PARTNER) trial analysis. Circulation 2013;127:2316-26.

14. Thaden JJ, Nkomo VT, Lee KJ, et al. Doppler Imaging in Aortic Stenosis: The Importance of the Nonapical Imaging Windows to Determine Severity in a Contemporary Cohort. J Am Soc Echocardiogr 2015;28:780-5.

15. Baumgartner H, Hung J, Bermejo J, et al.

Recommendations on the Echocardiographic Assessment of Aortic Valve Stenosis: A Focused Update from the European Association of Cardiovascular Imaging and the American Society of Echocardiography. J Am Soc Echocardiogr 2017;30:372-92.

16. Shiran A, Adawi S, Ganaeem M, et al. Accuracy and reproducibility of left ventricular outflow tract diameter measurement using transthoracic when compared with transesophageal echocardiography in systole and diastole. Eur J Echocardiogr 2009;10:319-24.

17. Saitoh T, Shiota M, Izumo M, et al. Comparison of left ventricular outflow geometry and aortic valve area in patients with aortic stenosis by 2-dimensional versus 3-dimensional echocardiography. Am J Cardiol 2012;109:1626-31.

18. Muraru D, Badano LP, Vannan M, et al. Assessment of aortic valve complex by three-dimensional echocardiography: a framework for its effective application in clinical practice. Eur Heart J Cardiovasc Imaging 2012;13:541-55.

19. Barbanti M, Yang TH, Rodes Cabau J, et al. Anatomical and procedural features associated with aortic root rupture during balloon-expandable transcatheter aortic valve replacement. Circulation 2013;128:244-53.

20. Athappan G, Patvardhan E, Tuzcu EM, et al. Incidence, predictors, and outcomes of aortic regurgitation after transcatheter aortic valve replacement: meta-analysis 
and systematic review of literature. J Am Coll Cardiol 2013;61:1585-95.

21. Détaint D, Lepage L, Himbert D, et al. Determinants of significant paravalvular regurgitation after transcatheter aortic valve: implantation impact of device and annulus discongruence. JACC Cardiovasc Interv 2009;2:821-7.

22. Ribeiro HB, Nombela-Franco L, Urena M, et al. Coronary obstruction following transcatheter aortic valve implantation: a systematic review. JACC Cardiovasc Interv 2013;6:452-61.

23. Piazza N, de Jaegere P, Schultz C, et al. Anatomy of the aortic valvar complex and its implications for transcatheter implantation of the aortic valve. Circ Cardiovasc Interv 2008;1:74-81.

24. Tzikas A, Schultz CJ, Piazza N, et al. Assessment of the aortic annulus by multislice computed tomography, contrast aortography, and trans-thoracic echocardiography in patients referred for transcatheter aortic valve implantation. Catheter Cardiovasc Interv 2011;77:868-75.

25. Koos R, Altiok E, Mahnken AH, et al. Evaluation of aortic root for definition of prosthesis size by magnetic resonance imaging and cardiac computed tomography: implications for transcatheter aortic valve implantation. Int J Cardiol 2012;158:353-8.

26. Altiok E, Hamada S, van Hall S, et al. Comparison of direct planimetry of mitral valve regurgitation orifice area by three-dimensional transesophageal echocardiography to effective regurgitant orifice area obtained by proximal flow convergence method and vena contracta area determined by color Doppler echocardiography. Am J Cardiol 2011;107:452-8.

27. Hahn RT, Pibarot P. Accurate Measurement of Left Ventricular Outflow Tract Diameter: Comment on the Updated Recommendations for the Echocardiographic Assessment of Aortic Valve Stenosis. J Am Soc Echocardiogr 2017;30:1038-41.

28. Jilaihawi H, Kashif M, Fontana G, et al. Cross-sectional computed tomographic assessment improves accuracy of aortic annular sizing for transcatheter aortic valve replacement and reduces the incidence of paravalvular aortic regurgitation. J Am Coll Cardiol 2012;59:1275-86.

29. Gurvitch R, Webb JG, Yuan R, et al. Aortic annulus diameter determination by multidetector computed tomography: reproducibility, applicability, and implications for transcatheter aortic valve implantation. JACC Cardiovasc Interv 2011;4:1235-45.

30. Khalique OK, Kodali SK, Paradis JM, et al. Aortic annular sizing using a novel 3-dimensional echocardiographic method: use and comparison with cardiac computed tomography. Circ Cardiovasc Imaging 2014;7:155-63.

31. Ng AC, Delgado V, van der Kley F, et al. Comparison of aortic root dimensions and geometries before and after transcatheter aortic valve implantation by 2 - and 3-dimensional transesophageal echocardiography and multislice computed tomography. Circ Cardiovasc Imaging 2010;3:94-102.

32. Oshita C, Murata K, Wada Y, et al. Assessment of the aortic valve annular geometry by real-time threedimensional transthoracic echocardiography: comparison with two-dimensional transthoracic echocardiography and multidetector computed tomography. J Echocardiogr 2014;12:24-30.

33. Khalique OK, Hahn RT, Gada H, et al. Quantity and location of aortic valve complex calcification predicts severity and location of paravalvular regurgitation and frequency of post-dilation after balloon-expandable transcatheter aortic valve replacement. JACC Cardiovasc Interv 2014;7:885-94.

34. Ewe SH, Ng AC, Schuijf JD, et al. Location and severity of aortic valve calcium and implications for aortic regurgitation after transcatheter aortic valve implantation. Am J Cardiol 2011;108:1470-7.

35. Watanabe Y, Lefevre T, Bouvier E, et al. Prognostic value of aortic root calcification volume on clinical outcomes after transcatheter balloon-expandable aortic valve implantation. Catheter Cardiovasc Interv 2015;86:1105-13.

36. Staubach S, Franke J, Gerckens U, et al. Impact of aortic valve calcification on the outcome of transcatheter aortic valve implantation: results from the prospective multicenter German TAVI registry. Catheter Cardiovasc Interv 2013;81:348-55.

37. Ribeiro HB, Webb JG, Makkar RR, et al. Predictive factors, management, and clinical outcomes of coronary obstruction following transcatheter aortic valve implantation: insights from a large multicenter registry. J Am Coll Cardiol 2013;62:1552-62.

38. Hayashida K, Bouvier E, Lefevre T, et al. Transcatheter aortic valve implantation for patients with severe bicuspid aortic valve stenosis. Circ Cardiovasc Interv 2013;6:284-91.

39. Yousef A, Simard T, Webb J, et al. Transcatheter aortic valve implantation in patients with bicuspid aortic valve: A patient level multi-center analysis. Int J Cardiol 2015;189:282-8.

40. Mylotte D, Lefevre T, Sondergaard L, et al. Transcatheter aortic valve replacement in bicuspid aortic valve disease. J Am Coll Cardiol 2014;64:2330-9. 
41. Nombela-Franco L, Ribeiro HB, Urena M, et al. Significant mitral regurgitation left untreated at the time of aortic valve replacement: a comprehensive review of a frequent entity in the transcatheter aortic valve replacement era. J Am Coll Cardiol 2014;63:2643-58.

42. Toggweiler S, Boone RH, Rodes-Cabau J, et al. Transcatheter aortic valve replacement: outcomes of patients with moderate or severe mitral regurgitation. J Am Coll Cardiol 2012;59:2068-74.

43. Bedogni F, Latib A, De Marco F, et al. Interplay between mitral regurgitation and transcatheter aortic valve replacement with the CoreValve Revalving System: a multicenter registry. Circulation 2013;128:2145-53.

44. Chakravarty T, Van Belle E, Jilaihawi H, et al. Metaanalysis of the impact of mitral regurgitation on outcomes after transcatheter aortic valve implantation. Am J Cardiol 2015;115:942-9.

45. Webb JG, Pasupati S, Humphries K, et al. Percutaneous transarterial aortic valve replacement in selected high-risk patients with aortic stenosis. Circulation 2007;116:755-63.

46. Gonçalves A, Marcos-Alberca P, Almeria C, et al. Acute left ventricle diastolic function improvement after transcatheter aortic valve implantation. Eur J Echocardiogr 2011;12:790-7.

47. Vizzardi E, D'Aloia A, Fiorina C, et al. Early regression of left ventricular mass associated with diastolic improvement after transcatheter aortic valve implantation. J Am Soc Echocardiogr 2012;25:1091-8.

48. Tarantini G, Buja P, Scognamiglio R, et al. Aortic valve replacement in severe aortic stenosis with left ventricular dysfunction: determinants of cardiac mortality and ventricular function recovery. Eur J Cardiothorac Surg 2003;24:879-85.

49. Pai RG, Varadarajan P, Razzouk A. Survival benefit of aortic valve replacement in patients with severe aortic stenosis with low ejection fraction and low gradient with normal ejection fraction. Ann Thorac Surg 2008;86:1781-9.

50. Passeri JJ, Elmariah S, Xu K, et al. Transcatheter aortic valve replacement and standard therapy in inoperable patients with aortic stenosis and low EF. Heart 2015;101:463-71.

51. Bach DS. Subvalvular left ventricular outflow obstruction for patients undergoing aortic valve replacement for aortic stenosis: echocardiographic recognition and identification of patients at risk. J Am Soc Echocardiogr 2005;18:1155-62.

52. Sherrid MV, Gunsburg DZ, Pearle G. Mid-systolic drop in left ventricular ejection velocity in obstructive hypertrophic cardiomyopathy--the lobster claw abnormality. J Am Soc
Echocardiogr 1997;10:707-12.

53. Takeda Y, Nakatani S, Kuratani T, et al. Systolic anterior motion of the mitral valve and severe mitral regurgitation immediately after transcatheter aortic valve replacement. J Echocardiogr 2012;10:143-5.

54. Smith CR, Leon MB, Mack MJ, et al. Transcatheter versus surgical aortic-valve replacement in high-risk patients. $\mathrm{N}$ Engl J Med 2011;364:2187-98.

55. Kodali SK, Williams MR, Smith CR, et al. Two-year outcomes after transcatheter or surgical aortic-valve replacement. N Engl J Med 2012;366:1686-95.

56. Zoghbi WA, Chambers JB, Dumesnil JG, et al. Recommendations for evaluation of prosthetic valves with echocardiography and doppler ultrasound: a report From the American Society of Echocardiography's Guidelines and Standards Committee and the Task Force on Prosthetic Valves, developed in conjunction with the American College of Cardiology Cardiovascular Imaging Committee, Cardiac Imaging Committee of the American Heart Association, the European Association of Echocardiography, a registered branch of the European Society of Cardiology, the Japanese Society of Echocardiography and the Canadian Society of Echocardiography, endorsed by the American College of Cardiology Foundation, American Heart Association, European Association of Echocardiography, a registered branch of the European Society of Cardiology, the Japanese Society of Echocardiography, and Canadian Society of Echocardiography. J Am Soc Echocardiogr 2009;22:975-1014; quiz 1082-4.

57. Leon MB, Piazza N, Nikolsky E, et al. Standardized endpoint definitions for Transcatheter Aortic Valve Implantation clinical trials: a consensus report from the Valve Academic Research Consortium. J Am Coll Cardiol 2011;57:253-69.

58. Colli A, D'Amico R, Kempfert J, et al. Transesophageal echocardiographic scoring for transcatheter aortic valve implantation: impact of aortic cusp calcification on postoperative aortic regurgitation. J Thorac Cardiovasc Surg 2011;142:1229-35.

59. Kappetein AP, Head SJ, Genereux P, et al. Updated standardized endpoint definitions for transcatheter aortic valve implantation: the Valve Academic Research Consortium-2 consensus document. J Am Coll Cardiol 2012;60:1438-54.

60. Brennan JM, Edwards FH, Zhao Y, et al. Long-term safety and effectiveness of mechanical versus biologic aortic valve prostheses in older patients: results from the Society 
of Thoracic Surgeons Adult Cardiac Surgery National Database. Circulation 2013;127:1647-55.

61. Tasca G, Brunelli F, Cirillo M, et al. Impact of valve prosthesis-patient mismatch on left ventricular mass regression following aortic valve replacement. Ann Thorac Surg 2005;79:505-10.

62. Kohsaka S, Mohan S, Virani S, et al. Prosthesis-patient mismatch affects long-term survival after mechanical valve replacement. J Thorac Cardiovasc Surg 2008;135:1076-80.

63. Hahn RT, Pibarot P, Stewart WJ, et al. Comparison of transcatheter and surgical aortic valve replacement in severe aortic stenosis: a longitudinal study of echocardiography parameters in cohort A of the PARTNER trial (placement of aortic transcatheter valves). J Am Coll Cardiol 2013;61:2514-21.

64. Pibarot P, Weissman NJ, Stewart WJ, et al. Incidence and sequelae of prosthesis-patient mismatch in transcatheter versus surgical valve replacement in high-risk patients with severe aortic stenosis: a PARTNER trial cohort--a analysis.
J Am Coll Cardiol 2014;64:1323-34.

65. Foroutan F, Guyatt GH, Otto CM, et al. Structural valve deterioration after transcatheter aortic valve implantation. Heart 2017;103:1899-905.

66. Amat-Santos IJ, Messika-Zeitoun D, Eltchaninoff H, et al. Infective endocarditis after transcatheter aortic valve implantation: results from a large multicenter registry. Circulation 2015;131:1566-74.

67. Makkar RR, Fontana G, Jilaihawi H, et al. Possible Subclinical Leaflet Thrombosis in Bioprosthetic Aortic Valves. N Engl J Med 2015;373:2015-24.

68. Yanagisawa R, Hayashida K, Yamada Y, et al. Incidence, Predictors, and Mid-Term Outcomes of Possible Leaflet Thrombosis After TAVR. JACC Cardiovasc Imaging 2016 8. pii: S1936-878X(16)30897-X.

69. Latib A, Naganuma T, Abdel-Wahab M, et al. Treatment and clinical outcomes of transcatheter heart valve thrombosis. Circ Cardiovasc Interv 2015;8. pii: e001779.
Cite this article as: Onishi T, Sengoku K, Ichibori Y, Mizote I, Maeda K, Kuratani T, Sawa Y, Sakata Y. The role of echocardiography in transcatheter aortic valve implantation. Cardiovasc Diagn Ther 2018;8(1):3-17. doi: 10.21037/ cdt.2018.01.06 